EDITOR'S
CHOICE

Radiology Department Papworth Hospital NHS Trust, Cambridge, UK

\section{Correspondence to} SK Agrawal, Radiology Department, Papworth Hospital NHS Trust, Papworth Everard, Cambridge CB23 3RE, UK : bobby.agrawal@papworth.nhs.

Received 9 November 2009 Accepted 13 February 2010

\title{
Ischaemic heart disease assessment by cardiovascular magnetic resonance imaging
}

\author{
Vimal Raj, S K Agrawal
}

\begin{abstract}
Ischaemic heart disease (IHD) related mortality has been on the decline, although its prevalence has been on the rise since the late 1970s. One of the contributing factors to this decline has been improved diagnosis and therapeutic management. Every clinician seeks to answer four key questions while evaluating patients with suspected or known IHD: What is the global ventricular function? What is the regional ventricular function? Is the myocardium viable? What is the status of the coronary arteries? In the past decade cardiovascular magnetic resonance (CMR) imaging has emerged as an important clinical technique with the potential of answering all the pertinent questions in a single study. This has led to a significant increase in demand and utilisation of this modality. However, many clinicians are not well versed with this technology, its clinical utility, limitations and future prospects. With the increasing prevalence of IHD, CMR imaging is likely to be used more often in its diagnosis, prognostication and management. The review describes the basic principles and practical aspects of CMR imaging, and then discusses in detail the role of $\mathrm{CMR}$ in the diagnosis and management of $\mathrm{HD}$, its complications, and its utility in patients with acute myocardial infarction.
\end{abstract}

Ischaemic heart disease (IHD) is the most common cause of death in the UK, with one in five men and one in six women dying from the disease. Death rates from IHD in the UK have been declining since late $1970 \mathrm{~s},{ }^{1}$ although its prevalence is on the rise. This fall in death rate could be attributed to a multitude of causes including changing lifestyle, environmental factors, and advancements in health care. Cardiovascular magnetic resonance (CMR) imaging is one such advancement in the field of health care, which has emerged as an important clinical technique in the diagnosis, therapeutics and prognostication of IHD.

In assessing a patient with IHD every clinician seeks to answer four key questions as shown in box 1. In the past, non-invasive imaging of IHD included chest radiography, echocardiography (echo), and nuclear cardiology. These different imaging modalities were used in conjunction with each other to answer these specific questions, which led to inadvertent delays in management and had significant financial implications. Clinical emphasis should be on better patient selection and initiation of appropriate treatment. This can be achieved by CMR, which over the last decade has emerged as a test with the potential of providing all the above information in a single study. CMR, with its higher spatial resolution and excellent soft tissue contrast, can also provide valuable information that may not be available from other diagnostic tools. ${ }^{2}$ This has led to its increased clinical demand and utilisation worldwide. However, many clinicians are not well versed with this technology, its clinical utility and limitations. We describe the basic principles and practical aspects of CMR imaging, and then discuss in detail the role of CMR in the diagnosis and management of IHD, its complications, and its utility in patients with acute myocardial infarction.

\section{BASIC PRINCIPLES AND PRACTICAL ASPECTS OF CMR IMAGING}

Magnetic resonance imaging (MRI) is dependent on the magnetic properties of the spinning hydrogen nuclei found within water and fat molecules. The scanner itself is a large magnet and when a patient enters it, the inherent spinning hydrogen nuclei align either along or against the magnetic field of the scanner. This alignment is then disturbed by an extrinsic radiofrequency pulse, which is then turned off, allowing nuclei to return back to their initial state of alignment. The latter leads to release of a radio signal that is used to create the final image.

CMR can acquire both static and live cine images. To prevent blurring of the image, ECG gating is used. Static images are preferentially acquired during diastole while cine images are acquired during the entire cardiac cycle. Different pulse sequences are used in CMR; these can be broadly classified into dark blood and bright blood sequences based on the colour of fast flowing blood. Dark blood sequences are good for anatomic delineation of blood vessel lumen and cardiac chambers. $^{3} 4$ Bright blood sequences are used for live cine imaging of the heart. ${ }^{5}$ The standard protocol used for assessing IHD by CMR in our institution is shown in table 1 . Images are acquired in standard planes that closely mimic the planes used in echocardiography and are shown in figure 1 The duration of CMR scan depends on the clinical question being answered and varies from $30 \mathrm{~min}$ to an hour. Patients are supine on the table and are asked to hold their breath (7-20 s) while images are being acquired. Arrhythmias and inability to hold breath adequately can lead to a suboptimal study.

MRI is safe and no long-term ill effects have been demonstrated. One of the most important safety issues for CMR is to avoid ferromagnetic substances near the scanner, as they can become projectiles. Metallic implants such as hip prostheses, prosthetic heart valves, coronary stents and sternal sutures are not a contraindication since these are not ferromagnetic. Care is required in patients with many cerebrovascular clips and 
Box 1 A clinician seeks to answer these questions while assessing patients with ischaemic heart disease

- What is the global ventricular function?

- What is the regional ventricular function?

- Is the myocardium viable?

-What is the status of coronary arteries?

specialist advice is needed for such patients. Patients with pacemakers, implantable cardioverter defibrillators (ICDs), retained permanent pacemaker leads and other electronic implants are currently not scanned. ${ }^{2}$

\section{CMR IN THE DIAGNOSIS AND MANAGEMENT OF ISCHAEMIC HEART DISEASE}

Pathophysiology of myocardial ischaemia

Myocardium may exist in different physiological states (table 2). The most precise definition of infarction, and therefore loss of viability, is that myocyte death must have occurred. The presence or absence of cell death can be established by microscopy or histological stains. However, this is not obviously practical in a clinical setting. Consequently, several less precise definitions of viability have been developed that are based on parameters that are more easily measured in patients. ${ }^{6} 7$ All ischaemic events before cell death are, at least in principle, reversible by re-establishment of an adequate blood supply. Therefore, it is important to differentiate between viable and non-viable states as revascularisation of the former improves left ventricular (LV) function and long term survival, while revascularisation of non-viable myocardium is associated with increased mortality. $^{8-12}$ Subendocardium is most vulnerable from ischaemia and, depending on the extent and duration of ischaemia, myocardial
Table 1 Standard cardiovascular magnetic resonance (CMR) protocol used for assessing ischaemic heart disease (IHD) with their specific clinical utility

\begin{tabular}{|c|c|}
\hline $\begin{array}{l}\text { IHD assessment CMR protocol } \\
\text { sequences }\end{array}$ & Clinical utility \\
\hline $\begin{array}{l}\text { Static dark blood images of the thorax } \\
\text { (HASTE sequence) }\end{array}$ & $\begin{array}{l}\text { Demonstrate anatomy and extra } \\
\text { cardiac abnormalities }\end{array}$ \\
\hline $\begin{array}{l}\text { Bright blood cine images in the } \\
\text { following planes }\end{array}$ & $\begin{array}{l}\text { Assess global and regional ventricular } \\
\text { function. }\end{array}$ \\
\hline $\begin{array}{l}2 \text { Chamber (vertical long axis) } \\
4 \text { Chamber (horizontal long axis) } \\
3 \text { Chamber } \\
\text { Short axis (from base to apex } \\
\text { of heart) } \\
\text { Left ventricular outflow tract (LVOT) } \\
\text { Right ventricular outflow tract (RVOT) }\end{array}$ & $\begin{array}{l}\text { Demonstrate valvular dysfunction } \\
\text { Detect ventricular thrombus }\end{array}$ \\
\hline $\begin{array}{l}\text { Myocardial stress imaging: } \\
\text { Adenosine stress/rest perfusion or } \\
\text { Dobutamine stress wall motion } \\
\text { analysis } \\
\text { Post-gadolinium enhancement static images }\end{array}$ & $\begin{array}{l}\text { Myocardial perfusion analysis } \\
\text { Myocardial contractile reserve }\end{array}$ \\
\hline Early & $\begin{array}{l}\text { Detect ventricular thrombus and } \\
\text { microvascular obstruction }\end{array}$ \\
\hline Late & Demonstrate myocardial fibrosis \\
\hline $\begin{array}{l}\text { Flow studies in breath hold and free } \\
\text { breathing }\end{array}$ & Valvular functional analysis \\
\hline Coronary artery imaging & Assess origins and proximal course \\
\hline
\end{tabular}

necrosis can extend transmurally towards the epicardium in a distribution corresponding to the perfusion territory of the diseased vessel. ${ }^{13}$ Viable myocardium may be hypo-contractile in the setting of chronic myocardial hypoperfusion or acute myocardial ischaemia. Hibernating and stunned myocardium are terms used to describe this phenomenon of viable but dysfunctional myocardium. Stunned myocardium is usually seen in the setting of acute infarction and shows improved recovery in the long term.
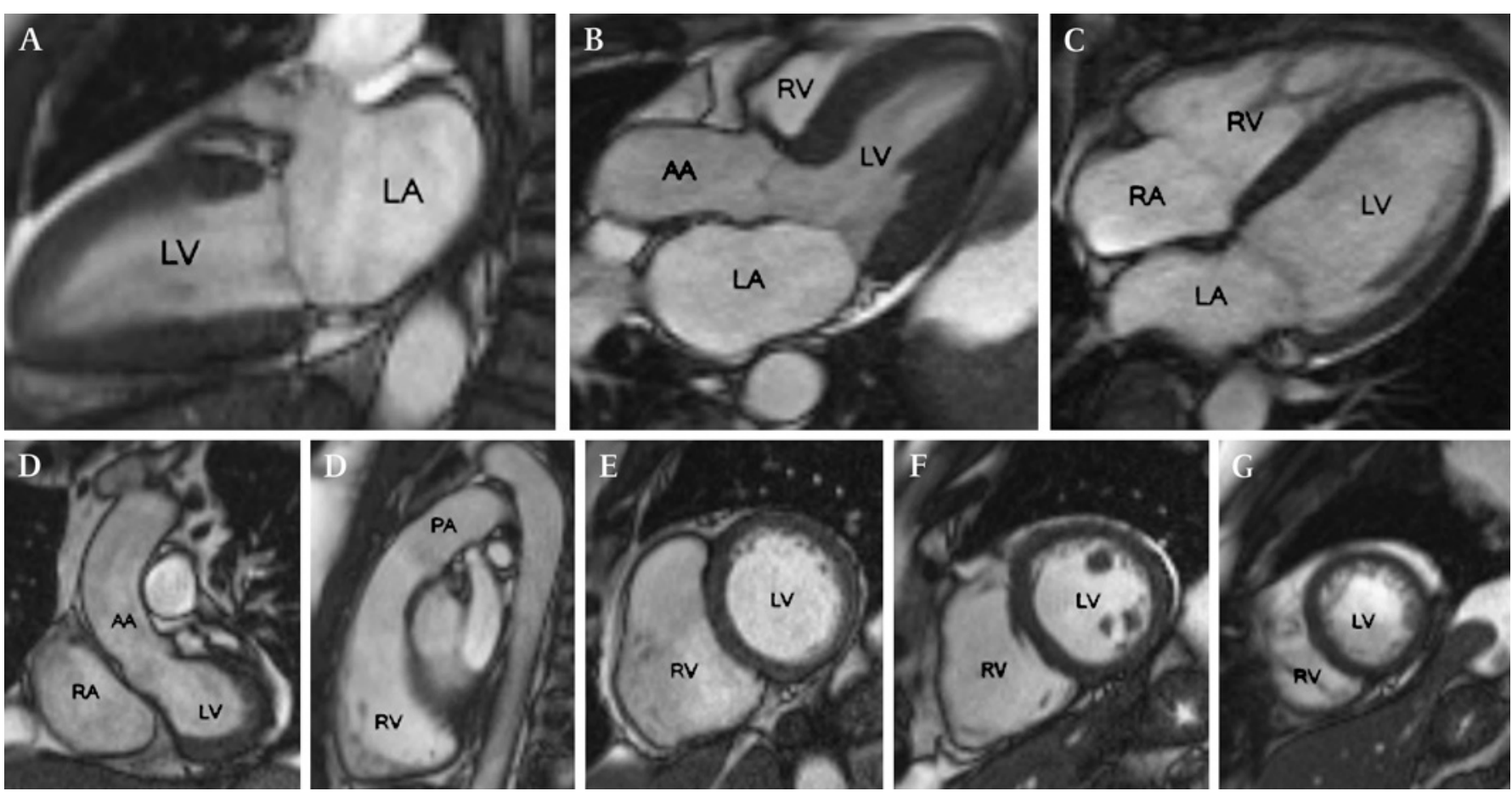

Figure 1 Standard planes of image acquisition on cardiovascular magnetic resonance (CMR) imaging. (A) Two chamber. (B) Three chamber. (C) Four chamber. (D) Left ventricular outflow tract (LVOT). (E) Right ventricular outflow tract (RVOT). (F) Sino-atrial (SA) base. (G) SA mid ventricle. (F) SA apical ventricle. AA, ascending aorta; LA, left atrium; LV, left ventricle; PA, pulmonary artery; RA, right atrium; RV, right ventricle. 
Table 2 Different physiological states of myocardium with their perfusion and contractile functionality

\begin{tabular}{lll}
\hline $\begin{array}{l}\text { Physiological state of } \\
\text { myocardium }\end{array}$ & $\begin{array}{l}\text { Contractile } \\
\text { function }\end{array}$ & Myocardial perfusion \\
\hline Viable: & Normal & $\begin{array}{l}\text { Normal } \\
\text { Transient decrease followed by } \\
\text { Normal } \\
\text { Stunned }\end{array}$ \\
Reduced & $\begin{array}{l}\text { recovery } \\
\text { Hibernating }\end{array}$ & Reduced \\
Non viable & None & None \\
\hline
\end{tabular}

\section{Clinical indications}

The clinical indications for performing CMR in patients suspected or known to have IHD are outlined in box $2 .^{2}$ As mentioned previously, in clinical practice it is not practical to assess myocardial viability by microscopy and histology. Information acquired from CMR can help in making this distinction between viable and non-viable myocardium non-invasively. The clinical indications of CMR in IHD are primarily aimed at answering the four critical questions outlined in box 1. Answers to these questions not only help in reaching the correct diagnosis but also help in streamlining the management and assessing the prognosis.

\section{Global ventricular function assessment by CMR}

In patients with IHD, quantification of LV functional parameters helps with risk stratification and guides clinical decision making. ${ }^{14}$ In the presence of both acute and chronic IHD, LV systolic function is a critical prognostic indicator with depressed function associated with poorer prognosis. ${ }^{8} 15$

Global LV function, myocardial mass and volume determination by CMR is performed using multiple cine short axis slices of the heart from the base to the apex. The endocardial and epicardial contours of the ventricle are drawn in automated analysis software. The LV volumes are then determined on the principles of Simpson's rule, whereby LV volumes are detected by summing the endocardial area within multiple short axis slices spanning the base to the apex and multiplying each area by the slice thickness (figure 2). ${ }^{16}$ This method of calculation does not require any assumptions of LV shape, allowing analysis of patients with cardiomyopathy and regional wall motion abnormality. ${ }^{16}$ LV ejection fraction (EF) is then calculated by

Box 2 Indications for cardiovascular magnetic resonance in ischaemic heart disease

1. Assessment of global ventricular (left and right) function and myocardial mass

2. Detection of coronary artery disease

Regional left ventricular function at rest and during dobutamine stress

Assessment of myocardial perfusion

Coronary angiography for anomalous coronary artery origins Coronary angiography for bypass graft patency (class II evidence)

Coronary angiography for coronary artery disease (class III evidence)

3. Acute and chronic myocardial infarction (MI) Detection and assessment $\rightarrow$ Myocardial viability Ventricular septal defect $\rightarrow$ Mitral regurgitation (acute MI) Ventricular thrombus subtracting the end systolic from the end diastolic volume (normal range $55-75 \%$ for males and females).

LV volumes and EF obtained by CMR are highly accurate, reproducible and well validated. The inter study reproducibility of CMR parameters is considerably superior to two dimensional and $M$ mode echocardiography, making it valuable for assessment of function and longitudinal follow-up of patients over time. $^{2}{ }^{16-21}$ In contrast to echocardiography, LVEF measurements are not impaired due to lack of suitable acoustic windows in some patients.

\section{Regional ventricular function assessment by CMR}

Regional ventricular function assessment by CMR includes regional wall motion abnormality (RWMA) and LV myocardial thickening. Regional functional analysis is vital as it can recognise areas of transmural chronic infarction which is characterised by severe myocardial thinning and lack of wall thickening in systole (figure 3). ${ }^{8} 22$ Multiple studies have shown that myocardial segments with an end diastolic wall thickness (EDWT) $<6 \mathrm{~mm}$ most likely represent transmural scar formation, and contractile function will not improve after revascularisation. However, an EDWT $>6 \mathrm{~mm}$ does not necessarily imply functional recovery after revascularisation. ${ }^{8} 23-25$

On CMR, RWMA is analysed on bright blood cine images acquired in multiple planes. For standardised assessment and documentation of myocardial analysis the 17- segment model of the American Heart Association is universally used (figure 4). ${ }^{26}$ A normal contractile pattern consists of endocardial inward movement and systolic wall thickening. RWMA is graded as: hypokinetic, when endocardial inward movement and systolic wall thickening are present but decreased; akinetic, when there is lack of endocardial inward movement and absent systolic wall thickening; and dyskinetic, when there is paradoxical systolic endocardial outward movement and/or systolic wall thinning. ${ }^{27}$ RWMA on CMR can be assessed both qualitatively (visually) and quantitatively. One of the quantitative analytic methods is myocardial tissue tagging where radiofrequency pulses are used to create regular dark bands (saturation bands) over the image. These bands form a diamond shape over the myocardium and will be distorted by myocardial contraction, while areas of akinesia or hypokinesia will have no or minimal distortion. 52829

Qualitative and quantitative RWMA analysis with CMR are both well validated. ${ }^{2}{ }^{29-31}$ As mentioned earlier, an EDWT $>6 \mathrm{~mm}$ on its own does not imply functional recovery after revascularisation and additional tests (stress and viability analysis) are required for predicting the outcome of these segments.

\section{Myocardial viability analysis by CMR}

Viable myocardium is characterised by the presence of living myocytes irrespective of contractile function or response to extrinsic stimuli. Hypocontractility in itself does not necessarily equate to non-viability and could be secondary to chronic myocardial hypoperfusion or acute myocardial ischaemia. Assessment of viability by CMR is based on demonstrating indirect signs of significant coronary artery disease, which include:

Myocardial contractile reserve by dobutamine stress study Adequate myocardial perfusion on adenosine/dipyridamole stress study

Intact myocyte cell membrane on delayed enhancement imaging.

\section{Myocardial contractile reserve by dobutamine stress study}

Bright blood cine CMR performed during dobutamine infusion is a well established technique for demonstrating ischaemia 
Figure 2 SA basal LV view in end diastole $(A)$ and end systole $(B)$ showing the endocardial (grey) and epicardial contours (white) around the myocardium to assess LV function. LV, left ventricle; RV, right ventricle.
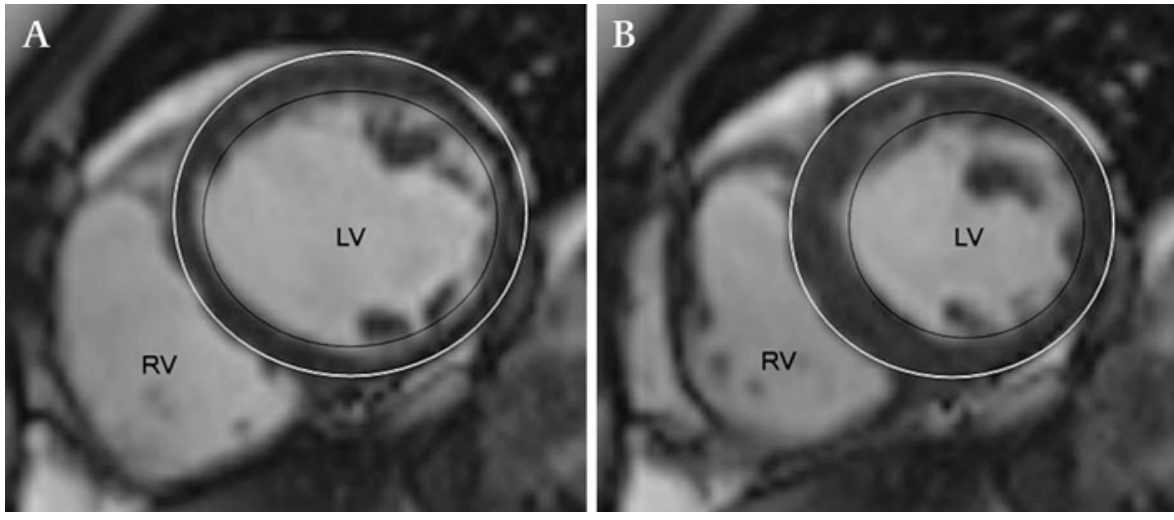

induced wall motion abnormality and may also be used to assess potential for contractile response to revascularisation in IHD. ${ }^{2} 32$

Dobutamine is a synthetic $\beta_{1}$ adrenergic catecholamine and has a positive inotropic effect. Dobutamine infusion leads to increased myocardial oxygen consumption which cannot be compensated by significantly occluded coronary arteries, leading to ischaemia and decreased contractility and thickening of the myocardium.

Dobutamine has a rapid onset of action and short half-life of about $120 \mathrm{~s}$, allowing gradual drug titration. To improve sensitivity, patients are asked to stop taking $\beta$-blockers $24-48 \mathrm{~h}$ before the test. ${ }^{32}$ Most side effects of dobutamine are mild (anxiety, palpitations, chest tightness, etc) and resolve spontaneously after termination of stress. If symptoms persist, side effects can be reversed by administration of a short acting $\beta$-blocker. Severe complications (ventricular tachycardia, ventricular fibrillation and myocardial infarction) are rare and can be seen in up to $0.3 \%$ of patients receiving high dose of dobutamine. ${ }^{33} 34$ Contraindications to dobutamine stress testing include acute coronary syndrome, severe aortic stenosis, hypertrophic obstructive cardiomyopathy, uncontrolled hypertension/ atrial fibrillation/heart failure, and known severe ventricular arrhythmias.

The study is performed by intravenous infusion of dobutamine in $3 \mathrm{~min}$ stages at incremental doses from $5-40 \mu \mathrm{g} / \mathrm{kg} /$ min under continuous monitoring of ECG, blood pressure (BP) and symptoms. If peak stress level, defined as target heart rate of [220-age] $\times 0.85$, is not achieved then additional fractioned atropine doses of $0.25 \mathrm{mg}$ every minute up to $1 \mathrm{mg}$ can be given. ${ }^{34}$ Selected cine images in both short and long axis are acquired at rest and at every incremental stage of stress. Images at different stages can then be compared side by side for the onset of new regional wall motion abnormalities.
Images can be analysed both visually and quantitatively. For standardised assessment and documentation findings are reported as per the 17-segment model. Dysfunctional but viable myocardium (hibernating and stunned) will show improvement of systolic contraction and wall thickening at low dose dobutamine infusion $(5-10 \mu \mathrm{g} / \mathrm{kg} / \mathrm{min})$. High dose dobutamine protocols (up to $40 \mu \mathrm{g} / \mathrm{kg} / \mathrm{min}$ ) are used to improve the sensitivity and to demonstrate the biphasic response: initial improvement of contractile function at low dose and worsening of contractile function at high dose, caused by stress induced ischaemia. ${ }^{25}$

Reported sensitivity and specificity of dobutamine CMR in predicting functional recovery ranges from $50-82 \%$ and $81-90 \%$, respectively. By using quantitative assessment methods the sensitivity and specificity can be as high as $89 \%$ and $93 \%$, respectively. ${ }^{8}$ Dobutamine CMR is superior to stress echocardiography due to higher quality imaging and lack of dependence on adequate ultrasound windows. ${ }^{2} 3536$ On the other hand, stress echocardiography is preferred by some clinicians in assessment of patients with concurrent mitral regurgitation or aortic stenosis. There is a low cardiovascular event rate when the dobutamine CMR is normal and a higher event rate in the presence of ischaemia. ${ }^{2}$

\section{Myocardial perfusion analysis by CMR}

Myocardial perfusion analysis is achieved by obtaining a live movie of the transit of contrast medium with the blood during its initial transit through the LV myocardium - in other words, it demonstrates the first pass perfusion of the myocardium. First pass perfusion of the LV myocardium is analysed at both peak stress and at rest. These are compared with each other to assess stress induced perfusion defects and ischaemia.

Adenosine is the most commonly used pharmacological agent for stress perfusion analysis. Adenosine is a vasodilator that
Figure 3 Basal LV view in end diastole (A) and end systole (B) showing thinning of the inferior myocardial wall (arrow) with lack of systolic thickening compared to the rest of the myocardium. LV, left ventricle; RV, right ventricle.
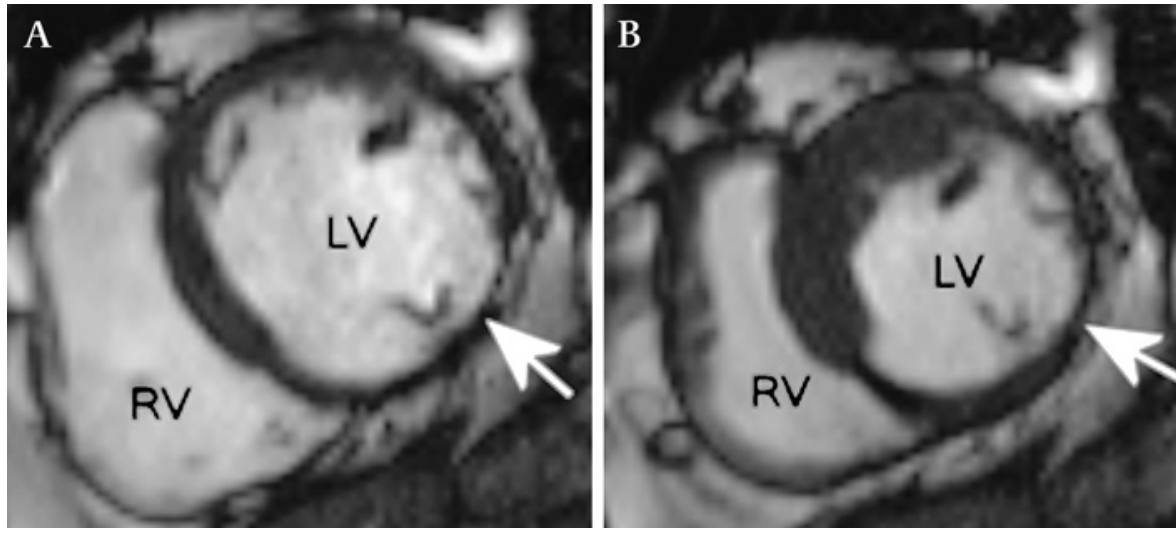

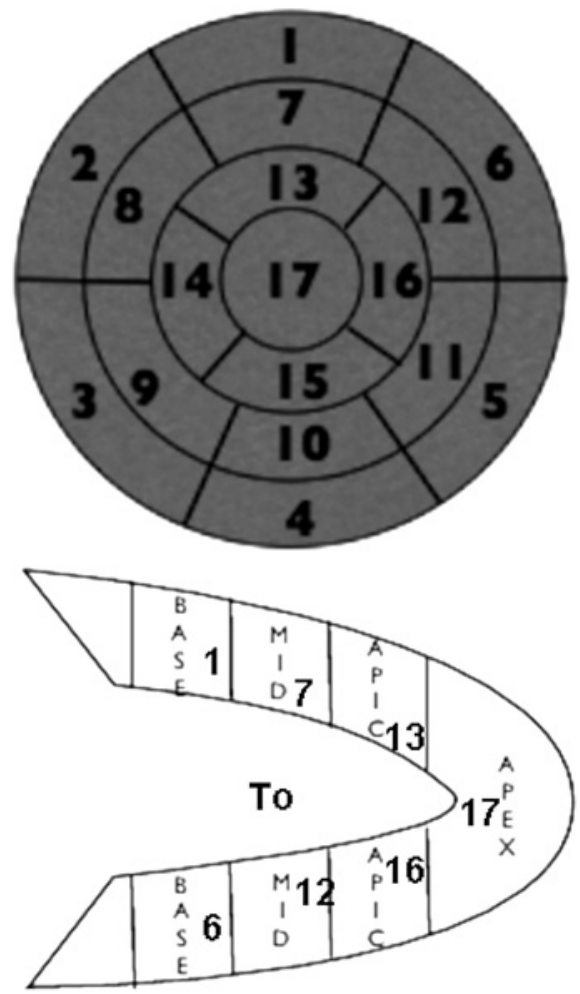

Figure 4 Seventeen segment model used for standard nomenclature, depicting the left ventricle basal, mid, apical cavity and apex distribution.

increases the myocardial blood flow by four- to fivefold downstream of a normal coronary artery. In a severely diseased artery there is no such increase in flow, as the capillaries are already maximally dilated, leading to delayed delivery of contrast which is seen as an area of hypo-enhancement or darkness.

Commonly adenosine is infused intravenously at the rate of $140 \mu \mathrm{g} / \mathrm{kg} / \mathrm{min}$ under continuous monitoring of blood pressure, ECG and symptoms. The available alternative agent is dipyridamole with a half-life of $30 \mathrm{~min}$, compared to $10 \mathrm{~s}$ of adenosine, making it difficult to control effectively. Patients usually experience shortness of breath, chest tightness, palpitations and a drop in blood pressure that resolve spontaneously after stopping adenosine. Adenosine is to be avoided in patients with severe asthma, high degree atrioventricular (AV) block, significant valvular stenosis and patients on dipyridamole due to its potentiating effect. ${ }^{37}$

Adenosine is infused for at least 3 min to achieve peak vasodilatation. After this a bolus of gadolinium contrast agent $(0.1 \mathrm{mmol} / \mathrm{kg})$ is injected and the myocardium is imaged. Recurrent images are acquired at selected short and long axis planes on every heartbeat (for 50 beats) while patients are holding their breath. The infusion is then stopped and early post-gadolinium imaging performed to demonstrate microvascular obstruction, which is seen as areas of low signal in subendocardial distribution. Late gadolinium enhancement (LGE) imaging is then performed, and is discussed in detail later. About 20 min after the completion of the adenosine infusion, myocardial rest perfusion is performed in a similar way to the stress study but without adenosine infusion. Images can be assessed qualitatively by visual inspection or semi-quantitatively. Visual analysis is adequate in a clinical setting and characterises perfusion defects as reversible when present only at stress, and fixed when present in both stress and rest (figure 5).

CMR is now an attractive alternative to single photon emission computed tomography (SPECT) and positron emission
Figure 5 SA LV mid $(A, C)$ and apical cavity $(B, D)$ views in peak stress $(A, B)$ and rest $(C$, D). Note significant subendocardial stress induced perfusion defect (arrow) in the antero septal wall which completely resolves at rest. $\mathrm{LV}$, left ventricle; RV, right ventricle.
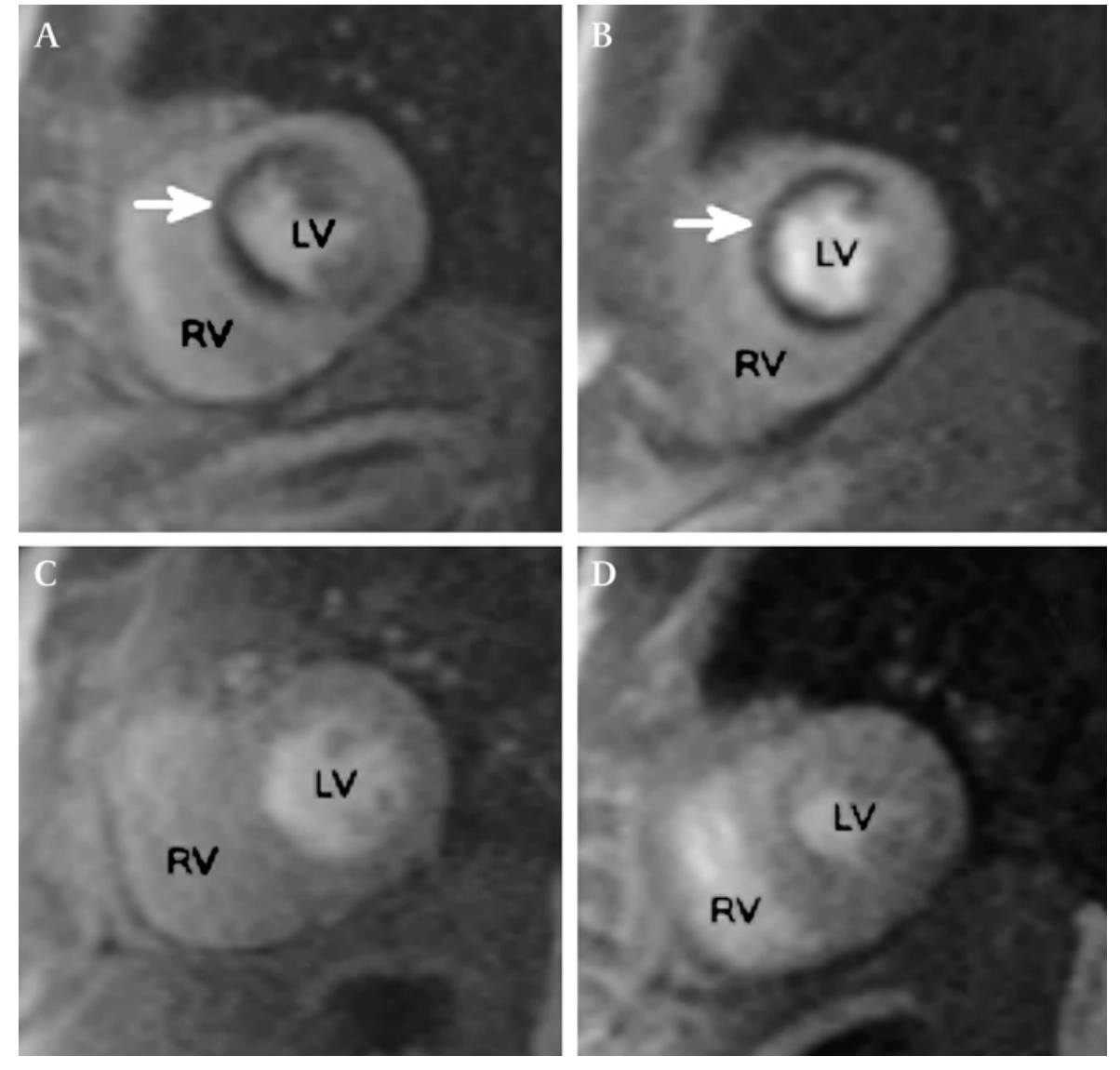
tomography (PET) myocardial perfusion imaging. This is due to improved subendocardial resolution, cost effectiveness, and lack of ionising radiation in CMR. Multiple trials comparing CMR with SPECT, PET and/or coronary angiography have shown positive results, with sensitivity and specificity of CMR in detecting perfusion defects of $87-90 \%$ and $85 \%$, respectively. ${ }^{38} 39$ CMR perfusion has also established its role in imaging patients with cardiac syndrome $\mathrm{X}$, wherein one has typical anginal symptoms, abnormal exercise test, but a normal coronary angiography. It is possible to show a subendocardial perfusion defect associated with intense chest pain by CMR in patients with syndrome $\mathrm{X}^{40}$

\section{LGE imaging}

Late or delayed phase enhancement imaging after gadolinium injection is a simple technique that allows detection of myocardial infarction (MI) and assessment of myocardial viability. This is perhaps the most widely used and validated CMR technique for assessing viability. Evidence from many previous studies has shown that delayed hyperenhancement is observed in regions of myocardial irreversible injury. ${ }^{9}$ 41-43

Gadolinium is an extracellular CMR contrast agent and does not traverse intact cell membranes. The normal myocardium is tightly packed with muscle with minimal extracellular space and has a uniform low signal. In areas of infarction, the extracellular compartment is expanded and washout of contrast from these areas is slow. This leads to a higher concentration of contrast which is seen as a bright signal. ${ }^{2}$ With its high spatial resolution $(1.2 \times 1.2 \mathrm{~mm})$ it is possible to determine the transmural distribution of MI on CMR (figure 6). Gadolinium has a good general safety profile, but needs to be used with caution in patients with impaired renal function and is contraindicated in patients with a glomerular filtration rate (GFR) $<30 \mathrm{ml} / \mathrm{min} /$ $1.73 \mathrm{~m}^{2}$ as it can induce nephrogenic systemic fibrosis (NSF). NSF is typically seen in patients with end stage renal disease and most are on regular dialysis treatment. It begins with subacute swelling of distal parts of the extremities followed during subsequent weeks by severe skin induration and sometimes anatomical extension. Skin induration may be aggressive and associated with constant pain while in some cases it can lead to serious disability requiring use of a wheelchair. NSF can also extend to involve several organs such as the liver, lungs and heart which may explain suspected increased mortality in these

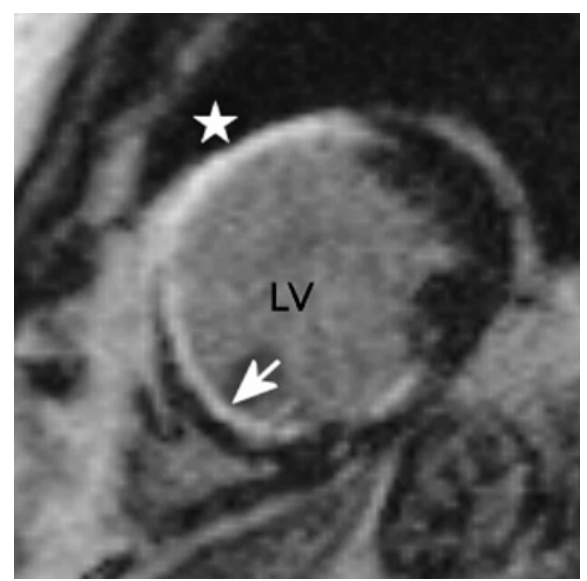

Figure 6 Late gadolinium enhancement SA mid cavity view showing, transmural enhancement (infarction) of the anteroseptal wall (star) and up to $50 \%$ subendocardial enhancement of the infero septum (arrow). LV, left ventricle. patients. ${ }^{44} 45$ There is no universal consensus about the pathophysiology of NSF, but the working hypothesis is that in patients with end stage renal failure there is delayed or no excretion of free gadolinium, which is extremely toxic. This free gadolinium is thought to deposit in the tissues and lead to NSF. $^{44} 46$

LGE imaging is typically performed $5-10 \mathrm{~min}$ after injection of gadolinium and static images are acquired in long axis and short axis plane covering the whole myocardium. To improve sensitivity and specificity of detecting hyperenhancement the signal from adjoining normal myocardium is nulled by using a special inversion recovery sequence.

Abnormal enhancement caused by IHD should be limited to a vascular territory and subendocardium is the most vulnerable part of the myocardium. LGE by CMR is highly sensitive and can identify abnormal enhancement even when wall motion and perfusion by SPECT are normal. ${ }^{47}$ LGE by CMR has been tested for predicting viability, and when the transmural extent of infarction/hyperenhancement is $<50 \%$ the likelihood of functional recovery in acute MI or with bypass surgery is good. ${ }^{48-50}$ Identification of infarcted myocardium is also important because it provides a substrate for ventricular tachyarrhythmias. The technique is reproducible, ${ }^{51}$ superior to SPECT, and excellent when directly compared with PET. ${ }^{2}$ 51-54

Myocardial hyperenhancement outlines areas of myocardial fibrosis, which is a final common pathway for myocardial injury. Therefore it can be seen in other conditions affecting the myocardium, although the pattern of hyperenhancement is different. In contrast to subendocardial/transmural enhancement seen in IHD, patients with sarcoidosis and dilated cardiomyopathy tend to have mid wall enhancement, while patients with hypertrophic cardiomyopathy have patchy enhancement not confined to a vascular territory. Myocarditis on the other hand can have either epicardial or mid myocardial hyperenhancement (figure 7).

Comprehensive evaluation of viability by CMR is performed differently at different centres. LGE imaging tends to form the core of viability analysis and can be used on its own or in combination with either dobutamine or adenosine stress analysis. The former has the advantage of being quick, safe and not requiring specialist supervision. However, addition of one of the stress protocols is thought to improve the specificity of the viability analysis. The choice between the two stress protocols is dependent on user experience, patient factors (for example, severe asthma is a contraindication for adenosine), and local guidelines. In our centre, apart from LGE, we reserve the use of adenosine stress study in patients with suspected or known single vessel coronary artery disease, whereas double or triple vessel disease assessment is performed by dobutamine stress study. This is to prevent false negative first pass perfusion analysis secondary to multivessel balanced myocardial ischaemia.

\section{Coronary artery imaging and CMR}

CMR at present has a limited role in coronary artery imaging. This is mainly due to the small size, tortuosity and constant movement of the coronary arteries with every heartbeat and breath. Multidetector computed tomography (MDCT) remains the forerunner in non-invasive assessment of coronary vessels because of its superior spatial resolution $(0.5-0.6 \mathrm{~mm})$ and shorter breath hold. Emphasis has therefore been on depicting the functional impact of coronary artery disease in the form of regional wall motion abnormality or perfusion defects. Currently coronary artery imaging by CMR can be performed in 


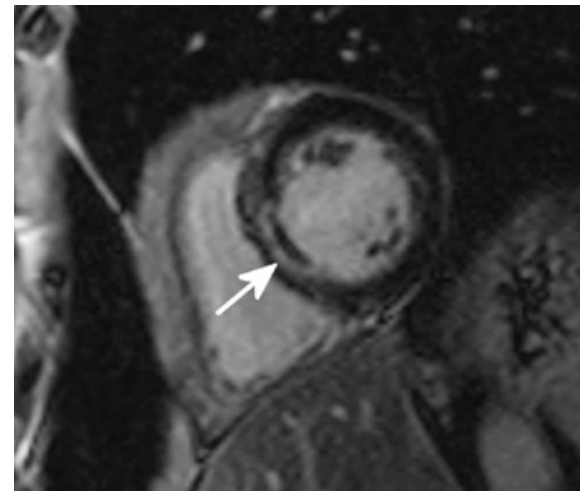

Figure 7 Late gadolinium enhancement SA mid cavity view showing septal mid wall enhancement (arrow). Clinical presentation and follow up imaging confirmed this to be myocarditis.

both breath-hold and free breathing states. The latter uses navigator technology where artefact secondary to breathing is nulled by tracking the movement of the diaphragm during image acquisition.

In clinical practice CMR coronary artery imaging is currently limited to assessing the anomalous origin and proximal course of the vessels (figure 8). This is usually performed after coronary angiography to ensure that the proximal portion dose not have a malignant course between the pulmonary artery and the aorta. $^{2} 5556$ There have been further promising studies assessing the role of CMR in imaging coronary artery disease and have shown a $81 \%$ negative predictive value in excluding multivessel proximal disease. ${ }^{57}$ However, assessment of severity of stenosis and distal vessels remains a technological challenge.

\section{CMR and complications of IHD}

CMR plays a vital role in assessment of complications secondary to both acute and chronic IHD. CMR in especially helpful in patients suspected to have ventricular thrombus, mitral regurgitation and myocardial rupture secondary to IHD. Ventricular thrombi can be detected during early and late phase gadolinium enhancement and usually require follow-up imaging to assess complete resolution following treatment. As imaging in multiple planes is possible with CMR, detection of septal or free wall rupture can be easily made. In cases of ventricular septal defect,

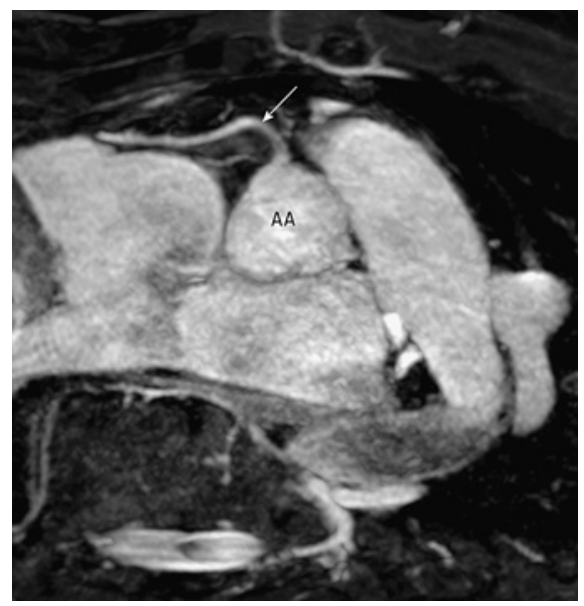

Figure 8 Axial (navigator) reformatted image showing normal origin and proximal course of the right coronary artery (arrow) from the aortic root $(A A)$. $A A$, ascending aorta.

\section{Key learning points}

With increasing prevalence of IHD there is increased demand and utilisation of CMR imaging in patients with IHD.

- CMR on its own allows comprehensive assessment of patients with IHD by providing accurate data on global and regional ventricular function.

- CMR can accurately distinguish viable from non-viable myocardium and can also demonstrate subendocardial ischaemia at a early stage.

- CMR has a limited role in coronary artery imaging.

- CMR also helps in surgical planning and prognosticating patients with IHD.

CMR not only helps in accurate quantification of interventricular shunt but is also useful in surgical planning. Mitral valve incompetence is a known complication of IHD. Along with LV functional analysis, CMR can provide important data on the amount of mitral regurgitation which will also guide further management.

\section{CMR imaging of acute MI}

CMR allows complete and accurate assessment of LV status in patients with acute MI, and contrast enhanced imaging visualises total infarct size and microvascular obstruction (MVO) in vivo. Despite successful recanalisation of infarct related artery by percutaneous coronary intervention, perfusion of the ischaemic myocardium is not completely restored in up to $30 \%$ of patients due to MVO. This is known to be a predictor of adverse events, higher incidence of ventricular remodelling, congestive heart failure, and death. The role of CMR in acute MI is currently limited to monitor patients after acute myocardial infarction in clinical trials. Because of its capacity to assess global/regional ventricular function, size and transmurality of infarction and $\mathrm{MVO}$ in one single examination, CMR is expected to become an important imaging modality in the evaluation of patients with acute MI. ${ }^{58}$

\section{SUMMARY}

CMR on its own allows comprehensive assessment of patients with IHD. Patients with poor LV function have a worse prognosis and CMR is the current gold standard imaging modality for assessment of ventricular function. Viability analysis is vital as revascularisation of non-viable myocardium and medical management of viable myocardium are associated with poor outcome. CMR can accurately distinguish viable from non-viable myocardium and demonstrate subendocardial ischaemia even before there is perfusion abnormalities on SPECT. The current role of CMR in coronary artery imaging is

\section{Current research questions}

- Improved myocardial perfusion analysis on 3 Tesla CMR scanners.

- Technological improvement in spatial resolution and ECG/ respiratory gating to improve coronary artery imaging on CMR.

- Role of CMR in evaluation of syndrome X. 


\section{Key references}

- Pennell DJ, Sechtem UP, Higgins CB, et al. Clinical indications for cardiovascular magnetic resonance (CMR): Consensus Panel report. Eur Heart J 2004;25:1940-65.

- Allman KC, Shaw LJ, Hachamovitch R, et al. Myocardial viability testing and impact of revascularization on prognosis in patients with coronary artery disease and left ventricular dysfunction: a meta-analysis. $J A m$ Coll Cardiol 2002;39:1151-8.

- Cerqueira MD, Weissman NJ, Dilsizian V, et al. Standardized myocardial segmentation and nomenclature for tomographic imaging of the heart: a statement for healthcare professionals from the Cardiac Imaging Committee of the Council on Clinical Cardiology of the American Heart Association. Circulation 2002;105:539-42.

- Gerber BL, Garot J, Bluemke DA, et al. Accuracy of contrastenhanced magnetic resonance imaging in predicting improvement of regional myocardial function in patients after acute myocardial infarction. Circulation 2002;106:1083-9.

- Kim RJ, Wu E, Rafael A, et al. The use of contrast enhanced magnetic resonance imaging to identify reversible myocardial dysfunction. N Engl J Med 2000;343:1445-53.

limited to assessment of the origin and proximal course of the vessels. From the available evidence and clinical practice, CMR might therefore be regarded as a 'one stop shop' for assessment of IHD.

\section{MULTIPLE CHOICE QUESTIONS (TRUE (T)/FALSE (F); ANSWERS AFTER REFERENCES)}

\section{Question 1:}

A. Ischaemic heart disease is the leading cause of death in UK

B. IHD related mortality is on the increase

C. Patients with permanent pacemakers cannot have CMR

D. CMR contrast agent contains gadolinium

E. Dobutamine is a negative inotropic agent

\section{Question 2:}

A. Left ventricular volumes and ejection fraction calculated by CMR are not reliable

B. There is a good likelihood of functional recovery in a myocardium which has $<50 \%$ subendocardial infarction on CMR

C. Subendocardium is the most viable part of myocardium to ischaemia

D. LGE is only seen in patients with myocardial infarction

E. NSF is usually seen in patients with good renal function

\section{Question 3:}

A. Hypocontractile myocardium is always non-viable

B. CMR is the imaging modality of choice for non-invasive assessment of coronary artery disease

C. CMR does not involve any radiation exposure to the patient

D. Adenosine used in myocardial stress test is a vasoconstrictor

E. Stunned myocardium is seen in the setting of acute myocardial infarction

\section{Question 4:}

A. Assessment of myocardial mass is a recognised indication for CMR

B. Depressed LV systolic function is a marker of poor prognosis
C. For standardised analysis and documentation, the 19-segment model of the American Heart Association is universally utilised

D. Regional wall motion abnormality on CMR can be analysed both quantitatively and qualitatively

E. Dobutamine has a short half life of $120 \mathrm{~min}$

\section{Question 5:}

A. Viable myocardium will show improvement in contractility at low dose dobutamine stress

B. Adenosine has a half life of $10 \mathrm{~s}$

C. Adenosine is contraindicated in patients with sever asthma

D. Coronary artery imaging by CMR can be performed under free breathing

E. ECG gating is not necessary in CMR

Competing interests None.

Provenance and peer review Not commissioned; not externally peer reviewed.

\section{REFERENCES}

1. Allender S, Peto V, Scarborough P, et al. Coronary heart disease statistics 2007. 2007 edition.

2. Pennell DJ, Sechtem UP, Higgins CB, et al. Clinical indications for cardiovascular magnetic resonance (CMR): Consensus Panel report. Eur Heart J 2004;25:1940-65.

3. Atkinson D, Teresi L. Magnetic resonance angiography. Magn Reson 0 1994;10:149-72

4. Finn JP, Edelman RR. Black-blood and segmented k-space magnetic resonance angiography. Magn Reson Imaging Clin N Am 1993;1:349-57.

5. Mehdi P-A, Fernando RG, Jeffrey JB, et al. Performing cardiac MR imaging: an overview. Magnetic resonance imaging clinics of North America 2003;11:1-18.

6. Jonathan WW, Igor K, Robert MJ. MRI for the Assessment of Myocardial Viability. Magnetic resonance imaging clinics of North America 2007;15:505-25.

7. Mahrholdt H, Klem I, Sechtem U. Cardiovascular MRI for detection of myocardial viability and ischaemia. Heart (British Cardiac Society) 2007 Date created: 2006/12/ 15/ Date completed 2007;93:122-9.

8. Schmidt A, Wu KC. MRI Assessment of Myocardial Viability. Seminars in Ultrasound, CT, and MRI 2006;27:11-19.

9. Wagner A, Mahrholdt $H$, Sechtem U, et al. MR imaging of myocardial perfusion and viability. Magnetic resonance imaging clinics of North America 2003:11:49-66.

10. Allman KC, Shaw LJ, Hachamovitch R, et al. Myocardial viability testing and impact of revascularization on prognosis in patients with coronary artery disease and left ventricular dysfunction: a meta-analysis. J Am Coll Cardiol 2002;39:1151-8.

11. Mock MB, Ringqvist I, Fisher LD, et al. Survival of medically treated patients in the coronary artery surgery study (CASS) registry. Circulation 1982;66:562-8.

12. Hammermeister KE, DeRouen TA, Dodge HT. Variables predictive of survival in patients with coronary disease. Selection by univariate and multivariate analyses from the clinical, electrocardiographic, exercise, arteriographic, and quantitative angiographic evaluations. Circulation 1979;59:421-30.

13. Leong-Poi H, Rim SJ, Le DE, et al. Perfusion versus function: the ischemic cascade in demand ischemia: implications of single-vessel versus multivessel stenosis. Circulation 2002;105:987-92.

14. Rackley CE, Russell RO Jr. Left ventricular function in acute and chronic coronary artery disease. Annu Rev Med 1975;26:105-20.

15. Emond M, Mock MB, Davis KB, et al. Long-term survival of medically treated patients in the Coronary Artery Surgery Study (CASS) Registry. Circulation 1994; $90: 2645-57$.

16. Walsh TF, Hundley WG. Assessment of Ventricular Function with Cardiovascular Magnetic Resonance. Magnetic Resonance Imaging Clinics of North America 2007; 15:487-504.

17. Bottini PB, Carr AA, Prisant $L M$, et al. Magnetic resonance imaging compared to echocardiography to assess left ventricular mass in the hypertensive patient. $A m J$ Hypertens 1995;8:221-8.

18. Grothues $\mathbf{F}$, Moon JC, Bellenger NG, et al. Interstudy reproducibility of right ventricular volumes, function, and mass with cardiovascular magnetic resonance. Am Heart J 2004;147:218-23.

19. Myerson SG, Bellenger NG, Pennell DJ. Assessment of left ventricular mass by cardiovascular magnetic resonance. Hypertension 2002;39:750-5.

20. Pattynama PM, Lamb HJ, van der Velde EA, et al. Left ventricular measurements with cine and spin-echo MR imaging: a study of reproducibility with variance component analysis. Radiology 1993;187:261-8.

21. Stratemeier EJ, Thompson R, Brady TJ, et al. Ejection fraction determination by MR imaging: comparison with left ventricular angiography. Radiology 1986;158:775-7.

22. McNamara MT, Higgins CB. Magnetic resonance imaging of chronic myocardial infarcts in man. AJR Am J Roentgenol 1986;146:315-20.

23. Perrone-Filardi $\mathbf{P}$, Bacharach SL, Dilsizian $\mathbf{V}$, et al. Regional left ventricular wall thickening. Relation to regional uptake of 18fluorodeoxyglucose and 201TI in patients 
with chronic coronary artery disease and left ventricular dysfunction Circulation 1992;86:1125-37.

24. Baer FM, Voth E, Schneider CA, et al. Comparison of low-dose dobutaminegradient-echo magnetic resonance imaging and positron emission tomography with [18F]fluorodeoxyglucose in patients with chronic coronary artery disease. A functional and morphological approach to the detection of residual myocardial viability. Circulation 1995;91:1006-15.

25. Schinkel AF, Bax JJ, Poldermans D. Clinical assessment of myocardial hibernation. Heart 2005:91:111-17.

26. Cerqueira MD, Weissman NJ, Dilsizian V, et al. Standardized myocardial segmentation and nomenclature for tomographic imaging of the heart: a statement for healthcare professionals from the Cardiac Imaging Committee of the Council on Clinical Cardiology of the American Heart Association. Circulation 2002;105:539-42.

27. Geleijnse ML, Fioretti PM, Roelandt JR. Methodology, feasibility, safety and diagnostic accuracy of dobutamine stress echocardiography. J Am Coll Cardiol 1997;30:595-606

28. Masood S, Yang GZ, Pennell DJ, et al. Investigating intrinsic myocardial mechanics: the role of MR tagging, velocity phase mapping, and diffusion imaging. J Magn Reson Imaging 2000;12:873-83.

29. Rerkpattanapipat P, Mazur W, Link KM, et al. Assessment of cardiac function with MR imaging. Magnetic resonance imaging clinics of North America 2003;11:67-80.

30. Sechtem U, Sommerhoff BA, Markiewicz W, et al. Regional left ventricular wall thickening by magnetic resonance imaging: evaluation in normal persons and patients with global and regional dysfunction. Am J Cardiol 1987;59:145-51.

31. Underwood SR, Rees RS, Savage PE, et al. Assessment of regional left ventricular function by magnetic resonance. Br Heart J 1986;56:334-40.

32. Nagel E, Lorenz C, Baer F, et al. Stress cardiovascular magnetic resonance: consensus panel report. J Cardiovasc Magn Reson 2001;3:267-81.

33. Kuijpers D, Janssen CHC, van DPRM, et al. Dobutamine stress MRI Part I Safety and feasibility of dobutamine cardiovascular magnetic resonance in patients suspected of myocardial ischemia. Eur Radiol 2004:14:1823-8.

34. Strach K, Meyer C, Schild H, et al. Cardiac stress MR imaging with dobutamine. Eur Radiol 2006;16:2728-38

35. Nagel E, Lehmkuhl HB, Bocksch W, et al. Noninvasive diagnosis of ischemia-induced wall motion abnormalities with the use of high-dose dobutamine stress MRI: comparison with dobutamine stress echocardiography. Circulation 1999;99:763-70.

36. Nagel E, Lehmkuhl HB, Klein C, et al. Influence of image quality on the diagnostic accuracy of dobutamine stress magnetic resonance imaging in comparison with dobutamine stress echocardiography for the noninvasive detection of myocardia ischemia. Z Kardiol 1999;88:622-30.

37. Prasad SK, Lyne J, Chai $P$, et al. Role of CMR in assessment of myocardial perfusion. Eur Radiol 2005:15(Suppl. 2):B42-7.

38. Schwitter J, Nanz D, Kneifel S, et al. Assessment of Myocardial Perfusion in Coronary Artery Disease by Magnetic Resonance: A Comparison With Positron Emission Tomography and Coronary Angiography. Circulation 2001:103:2230-5.

39. Al-Saadi N, Nagel E, Gross M, et al. Noninvasive detection of myocardial ischemia from perfusion reserve based on cardiovascular magnetic resonance. Circulation 2000;101:1379-83.

40. Panting JR, Gatehouse PD, Yang GZ, et al. Abnormal subendocardial perfusion in cardiac syndrome $X$ detected by cardiovascular magnetic resonance imaging. N Engl J Med 2002;346:1948-53

41. Pereira RS, Prato FS, Sykes J, et al. Assessment of myocardial viability using MRI during a constant infusion of Gd-DTPA: further studies at early and late periods of reperfusion. Magn Reson Med 1999;42:60-8

42. Pereira RS, Prato FS, Wisenberg G, et al. The determination of myocardial viability using Gd-DTPA in a canine model of acute myocardial ischemia and reperfusion. Magn Reson Med 1996;36:684-93.
43. McNamara MT, Tscholakoff D, Revel D, et al. Differentiation of reversible and irreversible myocardial injury by MR imaging with and without gadolinium-DTPA. Radiology 1986;158:765-9.

44. Thomsen HS. Nephrogenic systemic fibrosis: A serious late adverse reaction to gadodiamide. Eur Radiol 2006;16:2619-21.

45. Cowper SE, Robin HS, Steinberg SM, et al. Scleromyxoedema-like cutaneous diseases in renal-dialysis patients. Lancet 2000;356:1000-1.

46. Shellock FG, Spinazzi A. MRI safety update 2008: part 1, MRI contrast agents and nephrogenic systemic fibrosis. AJR Am J Roentgenol 2008;191:1129-39.

47. Wagner A, Mahrholdt H, Holly TA, et al. Contrast-enhanced MRI and routine single photon emission computed tomography (SPECT) perfusion imaging for detection of subendocardial myocardial infarcts: an imaging study. Lancet 2003;361:374-9.

48. Ramani K, Judd RM, Holly TA, et al. Contrast magnetic resonance imaging in the assessment of myocardial viability in patients with stable coronary artery disease and left ventricular dysfunction. Circulation 1998:98:2687-94.

49. Gerber BL, Garot J, Bluemke DA, et al. Accuracy of contrast-enhanced magnetic resonance imaging in predicting improvement of regional myocardial function in patients after acute myocardial infarction. Circulation 2002;106:1083-9.

50. Kim RJ, Wu E, Rafael A, et al. The use of contrast enhanced magnetic resonance imaging to identify reversible myocardial dysfunction. $N$ Eng J Med 2000; 343:1445-53.

51. Mahrholdt H, Wagner A, Holly TA, et al. Reproducibility of Chronic Infarct Size Measurement by Contrast-Enhanced Magnetic Resonance Imaging. Circulation 2002; 106:2322-7

52. Kitagawa K, Sakuma H, Hirano T, et al. Acute myocardial infarction: myocardial viability assessment in patients early thereafter comparison of contrast-enhanced MR imaging with resting (201)TI SPECT. Single photon emission computed tomography. Radiology 2003:226:138-44.

53. Klein C, Nekolla SG, Bengel FM, et al. Assessment of Myocardial Viability With Contrast-Enhanced Magnetic Resonance Imaging: Comparison With Positron Emission Tomography 10.1161/hc0202.102123. Circulation 2002:105:162-7.

54. Kuhl HP, Beek AM, van der Weerdt AP, et al. Myocardial viability in chronic ischemic heart disease: comparison of contrast-enhanced magnetic resonance imaging with (18)F-fluorodeoxyglucose positron emission tomography. J Am Coll Cardiol 2003:41:1341-8

55. McConnell MV, Ganz P, Selwyn AP, et al. Identification of anomalous coronary arteries and their anatomic course by magnetic resonance coronary angiography. Circulation 1995:92:3158-62.

56. Post JC, van Rossum AC, Bronzwaer JG, et al. Magnetic resonance angiography of anomalous coronary arteries. A new gold standard for delineating the proximal course? Circulation 1995;92:3163-71.

57. Kim WY, Danias PG, Stuber M, et al. Coronary magnetic resonance angiography for the detection of coronary stenoses. N Engl J Med 2001;345:1863-9.

58. Nijveldt R, Beek AM, Hirsch A, et al. 'No-reflow' after acute myocardial infarction: direct visualisation of microvascular obstruction by gadolinium-enhanced CMR. Neth Heart J 2008;16:179-81.

\section{ANSWERS}

1. $A(T) ; B(F) ; C(T) ; D(T) ; E(F)$

2. $A(F) ; B(T) ; C(T) ; D(F) ; E(F)$

3. $A(F) ; B(F) ; C(T) ; D(F) ; E(T)$

4. $A(T) ; B(T) ; C(F) ; D(T) ; E(F)$

5. $A(T) ; B(T): C(T) ; D(T) ; E(F)$ 